\title{
Numerical Simulation of Liquid Metal Free-surface Flows in the Presence of a Uniform Static Magnetic Field
}

\author{
Toshio TAGAWA \\ Department of Aerospace Engineering, Tokyo Metropolitan University, Asahigaoka 6-6, Hino 191-0065 Japan. \\ E-mail: ttagawa@cc.tmit.ac.jp
}

(Received on September 8, 2006; accepted on December 9, 2006)

\begin{abstract}
Three-dimensional numerical simulations with a new-developed modeling of two-phase flow have been carried out for free-surface flows of an electric conducting fluid in the presence of a uniform static magnetic field. In this study, several examples such as collapsing liquid column, oscillating droplet in a non-gravitational field and falling droplet in a gravitational field are presented. The driving mechanisms of the flow in these problems are the gravity and the surface tension. The numerical results reveal that the induced electromagnetic force acts to dampen the electric conducting fluid flow efficiently when a uniform vertical magnetic field is applied while it acts to enforce a tendency of two-dimensional flow when a uniform horizontal magnetic field is applied.
\end{abstract}

KEY WORDS: free-surface flow; numerical simulation; magnetic field; surface tension.

\section{Introduction}

Liquid metal free-surface flows in the presence of an applied magnetic field have received considerable attention due to its relevance to electromagnetic processing of materials. For instance, Yasuda et al. ${ }^{1)}$ developed a notable technique of electromagnetic levitation by using simultaneous imposition of an alternating and a static magnetic field. In this technique, an alternating magnetic field is used to levitate the molten metal while an additional static magnetic field is used to stabilize the melt disturbance. They reported that owing to the imposition of the static magnetic, both the melt oscillation and convection were mostly suppressed while only the melt rotation parallel to the static magnetic field remained. This phenomenon includes various factors such as melt-gas free surface, surface tension, temperature gradient, electromagnetic force, etc and thus the reason of melt rotation has not been clarified yet. To elucidate such kinds of complex phenomena, numerical simulation may be a potential tool.

Concerning the numerical modeling of free-surface flows, various methods ${ }^{2-5}$ ) have been reported. The volume of fluid (VOF) method ${ }^{2}$ and the level set method $^{3)}$ are known to be the most popular ones among them. The difficulty in these methods lies in mass balance during computation especially for the case of large density difference between two phases as well as the accurate estimation of curvature. Furthermore, numerical results by using these methods in the presence of a magnetic field have been hardly reported. Therefore, a numerical modeling to simulate the free-surface flow in the presence of a magnetic field is desired. Hwang et al. ${ }^{6}$ carried out numerical calculation using BFC (Body Fitted Coordinate) for molten steel flow in an electromagnetic break system with considering meniscus shape. Gao et ll. $^{7)}$ simulated a movement of liquid metal droplet in a magnetic field using a VOF-CSF method. Tagawa $^{8)}$ performed numerical simulations of a falling droplet into a liquid layer in the presence of a uniform vertical magnetic field as a first-stage attempt. The numerical result was restricted to an axisymmetric case and the effect of induced electric field in the liquid metal was neglected. Besides, the density ratio between the liquid metal droplet and the atmospheric gas was up to 100 . Therefore in this paper, as a second-stage attempt, a simple numerical modeling of free-surface flows in an isothermal condition with higher density-ratio is presented. Furthermore, effect of a uniform static magnetic field on liquid metal free-surface flows is numerically investigated with the new-developed present modeling.

\section{Mathematical Modeling}

\subsection{Governing Equations}

In this paper, an incompressible, immiscible, two-phase flow modeling is introduced. It is assumed that both the liquid and gas are the incompressible Newtonian fluid, and both viscous dissipation and Joule heat are neglected. Therefore, we do not consider the energy equation, i.e. thermal convection due to buoyancy and thermo-capillary effects are neglected. The conservation of mass is generally written as follows:

$$
\frac{\partial \rho}{\partial t}+\vec{u} \cdot \vec{\nabla} \rho=-\rho(\vec{\nabla} \cdot \vec{u})
$$

This equation is valid not only for the compressible singlephase flows but also for the compressible two-phase flows. 
Since we limit ourselves to the analyses of incompressible two-phase flows, Eq. (1) can be split into two equations as follows:

$$
\begin{array}{r}
\vec{\nabla} \cdot \vec{u}=0 \\
\frac{\partial \rho}{\partial t}+\vec{u} \cdot \vec{\nabla} \rho=0
\end{array}
$$

The advection equation of density must be solved together with the continuity equation and also the following momentum equation (Navier-Stokes equation), which is

$$
\rho\left[\frac{\partial \vec{u}}{\partial t}+(\vec{u} \cdot \vec{\nabla}) \vec{u}\right]=-\vec{\nabla} p+\mu \nabla^{2} \vec{u}+\rho \vec{g}+\vec{f}_{\mathrm{em}}+\vec{f}_{\mathrm{sv}} \ldots
$$

where, $\vec{f}_{\text {em }}$ denotes the electromagnetic force induced in the liquid phase by applying a magnetic field, and $\vec{f}_{\text {sv }}$ is a modeled interfacial force acting per unit volume. The detailed explanation about $\vec{f}_{\text {sv }}$ will be made in the next subsection. In Eq. (4), the fluid density $\rho$ and the viscosity $\mu$ take quite different values depending on the fluid phases. Therefore, if we solve this momentum equation not only for the liquid and gas phases but also for the diffused interfacial phase simultaneously, it is necessary to define the viscosity $\mu$ within the transitional interface as a function of the density $\rho$, since this $\rho$ is known during the computation. One of simple expressions is

$$
\mu=\frac{\rho-\rho_{\mathrm{G}}}{\rho_{\mathrm{L}}-\rho_{\mathrm{G}}}\left(\mu_{\mathrm{L}}-\mu_{\mathrm{G}}\right)+\mu_{\mathrm{G}}
$$

here, $\rho_{\mathrm{L}}$ represents the liquid density, $\rho_{\mathrm{G}}$ the gas density, $\mu_{\mathrm{L}}$ the liquid viscosity, and $\mu_{\mathrm{G}}$ gas viscosity. Basically, Eqs. (2), (3), (4) and (5) govern the incompressible, immiscible, isothermal two-phase flows. However, in order to make computations more convenient and precisely, we introduce the index function $\phi$ as a color function which distinguishes whether the computational domain is the liquid phase, gas phase or transitional region of interface. The index function is dimensionless and defined as follows:

$$
\begin{cases}\phi=-0.5 & \text { for liquid phase } \\ -0.5<\phi<0.5 & \text { for transitional interface } \\ \phi=0.5 & \text { for gas phase }\end{cases}
$$

Besides, we establish a relationship between the density and the index function $\phi$ in the transitional interface as follows:

$$
\rho=\frac{1}{2}\left(\rho_{\mathrm{L}}+\rho_{\mathrm{G}}\right)-\left(\rho_{\mathrm{L}}-\rho_{\mathrm{G}}\right)\left[\phi+\frac{1}{2 \pi} \sin (2 \pi \phi)\right] \ldots
$$

By having introduced this kind of relationship, the following advection equation of $\phi$ substitutes for Eq. (3).

$$
\frac{\partial \phi}{\partial t}+\vec{u} \cdot \vec{\nabla} \phi=0
$$

Equation (8) is derived from Eqs. (3) and (7). One of the advantages to introduce Eq. (7) and to use Eq. (8) instead of Eq. (3) is to enable us to compute iso-density immiscible two-phase flows. The another advantage is to prevent the transitional interface from diffusing because the thickness of the interface estimated by $\rho$ is thinner than that by $\phi$. This idea is analogous to the tangent transformation reported by Yabe et al. in a text book. ${ }^{9)}$

\subsection{External Forces}

The electromagnetic force $\vec{f}_{\mathrm{em}}$ in Eq. (4) is given as

$$
\vec{f}_{\mathrm{em}}=\vec{j} \times \vec{b}
$$

here, $\vec{j}$ is the electric current density, and $\vec{b}$ is the magnetic flux density. In this paper, the applied magnetic field is assumed to be static and uniform. Since the induced magnetic field is negligibly small in comparison with the imposed one, the electric current density is governed by the Ohm's law together with the conservation of electric charge as follows:

$$
\begin{array}{r}
\vec{j}=\sigma(-\vec{\nabla} \psi+\vec{u} \times \vec{b}) \\
\vec{\nabla} \cdot \vec{j}=0
\end{array}
$$

Where, $\psi$ and $\sigma$ are the electric potential and the electric conductivity of fluid respectively. In order to implement analyses of two-phase flow in the presence of a magnetic field, the electric conductivity $\sigma$ should be also a function of $\phi$ in a similar way as follows:

$$
\sigma=\frac{1}{2}\left(\sigma_{\mathrm{L}}+\sigma_{\mathrm{G}}\right)-\left(\sigma_{\mathrm{L}}-\sigma_{\mathrm{G}}\right)\left[\phi+\frac{1}{2 \pi} \sin (2 \pi \phi)\right] \ldots . .
$$

Next, the interfacial surface force is discussed in a case of isothermal field. Originally, the interfacial tension is tangential surface force acting per unit length. If the interface has a local curvature, the normal component of the surface force appears although the tangential ones are canceled out in the isothermal condition. The normal surface force acting per unit area at the interface can be written with

$$
\vec{f}_{\mathrm{sa}}=\gamma \kappa \vec{e}_{\mathrm{n}}
$$

Here, $\gamma$ represents the interfacial tension, $\kappa$ the curvature and $\vec{e}_{\mathrm{n}}$ the unit vector normal to the interface. The surface force should be converted into a body force to be included in Eq. (4). Brackbill et al. derived such a body force (CSF model $)^{10)}$ and one of possible expressions using the fluid density is given and yielded from Eq. (7) as

$$
\vec{f}_{\mathrm{sv}}=\gamma \kappa \frac{\vec{\nabla} \rho}{\rho_{\mathrm{L}}-\rho_{\mathrm{G}}}=-\gamma \kappa[1+\cos (2 \pi \phi)] \vec{\nabla} \phi
$$

Here, the curvature $\kappa$ can be estimated by taking the divergence of the unit vector normal to the interface within the transitional region of interface. ${ }^{10)}$ It can be written as

$$
\kappa=\vec{\nabla} \cdot\left(\frac{\vec{\nabla} \phi}{|\vec{\nabla} \phi|}\right)
$$

Finally, Eq. (4) can be written with considering both the electromagnetic force and the interfacial body force. This gives

$$
\frac{\partial \vec{u}}{\partial t}+(\vec{u} \cdot \vec{\nabla}) \vec{u}=-\frac{1}{\rho} \vec{\nabla} p+\frac{\mu}{\rho} \nabla^{2} \vec{u}-g \vec{e}_{\mathrm{z}}+\frac{1}{\rho} \vec{j} \times \vec{b}
$$




$$
-\frac{\gamma}{\rho} \kappa[1+\cos (2 \pi \phi)] \vec{\nabla} \phi
$$

As recognized from Eq. (16), the interfacial body force is zero at any uniform phase. Therefore, the body force due to surface tension acts only within the interfacial phase in the direction normal to the interface depending on the value of curvature.

\subsection{Non-dimensional Equations}

The non-dimensional model equations for solving incompressible immiscible two-phase flows in the presence of a magnetic field are summarized as follows:

Continuity equation

$$
\vec{\nabla} \cdot \vec{U}=0
$$

Momentum equation

$$
\begin{aligned}
\frac{\partial \vec{U}}{\partial \tau}+ & (\vec{U} \cdot \vec{\nabla}) \vec{U} \\
= & -\frac{1}{\rho_{\phi}} \vec{\nabla} P+\frac{\mu_{\phi}}{\rho_{\phi}} \nabla^{2} \vec{U}-G \vec{e}_{\mathrm{z}}+\frac{\widehat{\mu}}{\rho_{\phi}} H a^{2} \vec{J} \times \vec{B} \\
& -\frac{\Gamma}{\rho_{\phi}} \vec{\nabla} \cdot(\vec{\nabla} \phi /|\vec{\nabla} \phi|)[1+\cos (2 \pi \phi)] \vec{\nabla} \phi \ldots \ldots . .(18)
\end{aligned}
$$

Advection equation

$$
\frac{\partial \phi}{\partial \tau}+\vec{U} \cdot \vec{\nabla} \phi=0
$$

Ohm's law

$$
\vec{J}=\sigma_{\phi}(-\vec{\nabla} \Psi+\vec{U} \times \vec{B})
$$

Conservation of electric charge

$$
\vec{\nabla} \cdot \vec{J}=0
$$

The dimensionless physical properties are defined as follows:

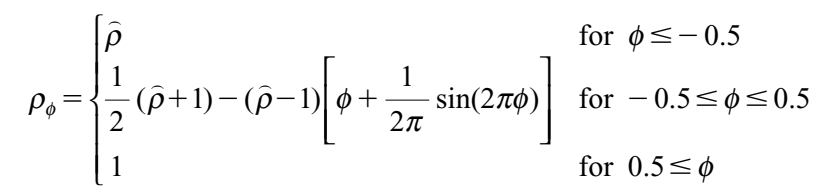

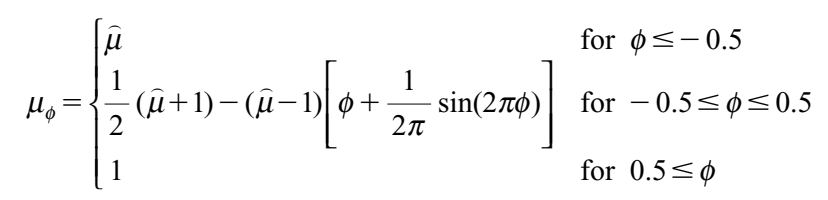

$$
\sigma_{\phi}= \begin{cases}1 & \text { for } \phi \leq-0.5 \\ \frac{1}{2}(1+\breve{\sigma})-(1-\breve{\sigma})\left[\phi+\frac{1}{2 \pi} \sin (2 \pi \phi)\right] & \text { for }-0.5 \leq \phi \leq 0.5 \\ \breve{\sigma}=0 & \text { for } 0.5 \leq \phi\end{cases}
$$

Where, the dimensionless variables are defined as follows:

$$
\begin{aligned}
& \vec{U}=\frac{\vec{u}}{\mu_{\mathrm{G}} /\left(\rho_{\mathrm{G}} d\right)}, \quad P=\frac{p}{\mu_{\mathrm{G}}^{2} /\left(\rho_{\mathrm{G}} d^{2}\right)}, \quad \tau=\frac{t}{\left(\rho_{\mathrm{G}} d^{2}\right) / \mu_{\mathrm{G}}}, \\
& \Psi=\frac{\psi}{\mu_{\mathrm{G}} b_{0} / \rho_{\mathrm{G}}}, \quad \vec{B}=\frac{\vec{b}}{b_{0}}, \quad \vec{J}=\frac{\vec{j}}{\left(\sigma_{\mathrm{L}} \mu_{\mathrm{G}} b_{0}\right) /\left(\rho_{\mathrm{G}} d\right)}, \\
& \rho_{\phi}=\frac{\rho}{\rho_{\mathrm{G}}}, \quad \mu_{\phi}=\frac{\mu}{\mu_{\mathrm{G}}}, \quad \sigma_{\phi}=\frac{\sigma}{\sigma_{\mathrm{L}}}
\end{aligned}
$$

The non-dimensional numbers are as follows:

$$
\begin{aligned}
& G=\frac{g \rho_{\mathrm{G}}^{2} d^{3}}{\mu_{\mathrm{G}}^{2}}, \quad H a=\sqrt{\frac{\sigma_{\mathrm{L}}}{\mu_{\mathrm{L}}}} b_{0} d, \quad \Gamma=\frac{\gamma \rho_{\mathrm{G}} d}{\mu_{\mathrm{G}}^{2}}, \\
& \hat{\rho}=\frac{\rho_{\mathrm{L}}}{\rho_{\mathrm{G}}}, \quad \bar{\mu}=\frac{\mu_{\mathrm{L}}}{\mu_{\mathrm{G}}}, \quad \check{\sigma}=\frac{\sigma_{\mathrm{G}}}{\sigma_{\mathrm{L}}}
\end{aligned}
$$

The parameters of the present computations are the Galilei number $(G)$, the Hartmann number $(\mathrm{Ha})$, the dimensionless tension number $(\Gamma)$, and three physical property ratios of density, viscosity and conductivity. The electric conductivity of air is nearly equal to zero and therefore the definition of conductivity ratio and the Hartmann number is defined as shown above. The characteristic length is indicated with $d$.

\subsection{Numerical Strategy}

The set of Eqs. (17) to (24) are transformed to finite difference equations on a staggered mesh system and solved numerically with a finite difference method. First, a predicted velocity field is computed with Eq. (18) together with Eqs. (22) and (23). Second, the velocity and pressure fields are corrected so as to satisfy Eq. (17) with an iterative procedure by the HSMAC method. ${ }^{11)}$ Third, the distribution of the index function (Eq. (19)) is calculated with the use of corrected velocity field. Fourth, a predicted current field is calculated with Eq. (20) together with Eq. (24). Fifth, the electric current and potential fields are corrected so as to satisfy Eq. (21) by use of the HSMAC method. ${ }^{11)}$

The inertial term in Eq. (18) and the advective term in Eq. (19) are approximated with a third-order upwind scheme (UTOPIA). ${ }^{12)}$ During the computation of Eq. (19), if there are portions greater than 0.5 or less than -0.5 for the value of index function, such portions are overwritten with the value of 0.5 or -0.5 respectively.

\section{Numerical Results}

In this section, several typical problems for free-surface flows are shown to evaluate the validity of the present mathematical modeling and to demonstrate MHD free-surface flows.

\subsection{Collapse of Water Column}

Figure 1 shows the schematic model for collapse of water column. A water column whose height is twice its width is released in a gravity field within a two-dimensional rectangular cavity. The relevant parameters for the simulation are the density ratio $\hat{\rho}$, viscosity ratio $\hat{\mu}$, Galilei number $G$, and tension number $\Gamma$. They are fixed at $\hat{\rho}=$ $800, \hat{\mu}=50, G=4.79 \times 10^{6}$ and $\Gamma=1.17 \times 10^{7}$, which correspond to real water-air free-surface flow with initial water 


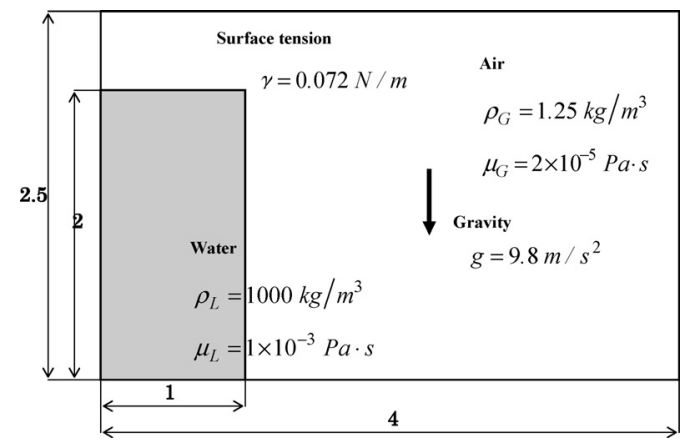

Fig. 1. Schematic model at the initial state of collapse of water column.
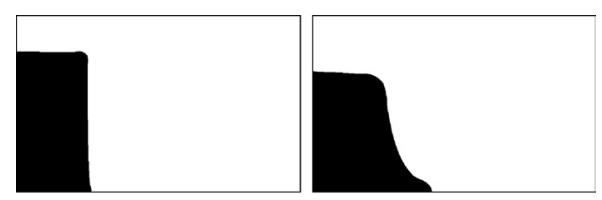

(a) $\tau=0.0001$

(b) $\tau=0.0004$

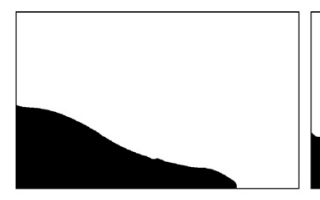

(c) $\tau=0.0008$

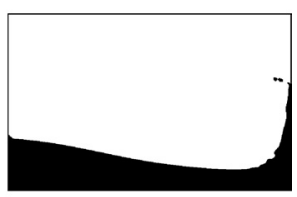

(d) $\tau=0.0012$

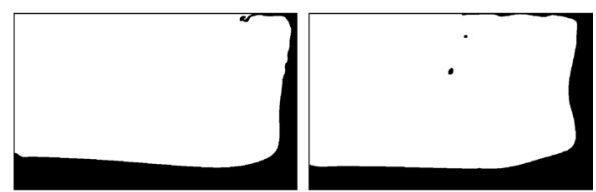

(e) $\tau=0.0016$

(f) $\tau=0.0020$

Fig. 2. Collapse of a water column for $G=4.79 \times 10^{6}, \Gamma=$ $1.17 \times 10^{7}$, density ratio $=800$, and viscosity ratio $=50$. The number of meshes is $400 \times 250$.

width $5 \mathrm{~cm}(a=0.05)$ and its height $10 \mathrm{~cm}$. All walls are assumed to be non-slip conditions. The number of meshes in this simulation is $400 \times 250$.

Figure 2 shows the evolution of the collapse of water column. The black part indicates $\phi \leq 0$ (water), while the white part $\phi>0$ (air). At the beginning of the computation (see (a)), an edge of water is rapidly rounded by the surface force acting at the interface between the air and water. Meanwhile, the bottom leading edge starts to move along the bottom non-slip wall. Although the driving forces in this collapse of water column are the gravity and surface tension, the latter effect is subordinate to the former one as recognized in the sequence of figures. The leading edge of water arrives at the right vertical wall at around $\tau=0.0010$ and then starts to ascend along the vertical wall. At $\tau=0.0020$, the water located near the right vertical wall is about to fall back.

In order to ensure the validity of this simulation, a dimensionless leading-edge location as a function of time is quantitatively compared with some previous experimental and numerical studies as shown in Fig. 3. The present result agrees with both the previous numerical simulation with the VOF method ${ }^{2)}$ and the previous experimental results referred in the recent paper. ${ }^{13)}$

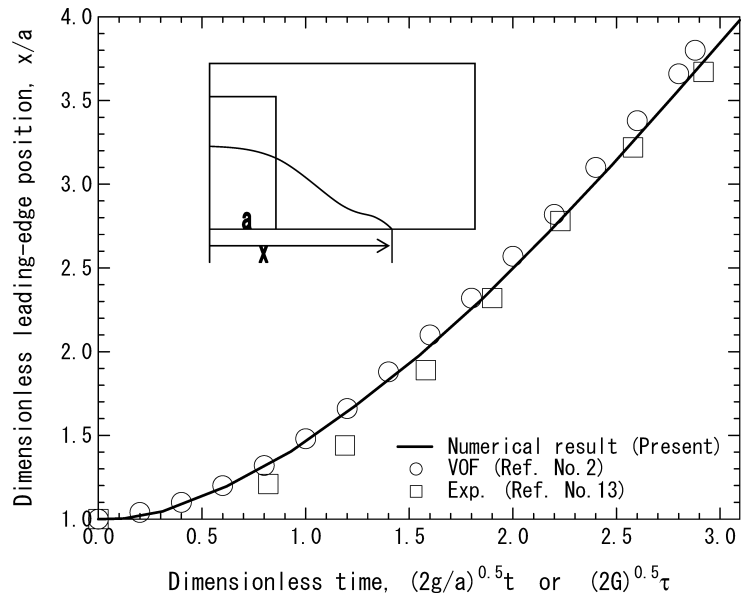

Fig. 3. Dimensionless leading-edge location as a function of time for $G=4.79 \times 10^{6}, \Gamma=1.17 \times 10^{7}$, density ratio $=800$, and viscosity ratio $=50$.

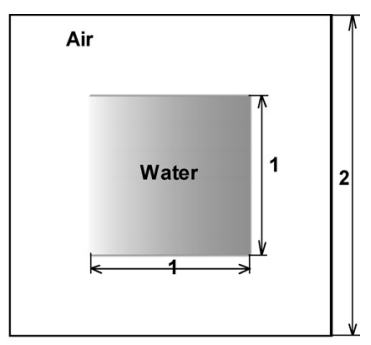

(b)

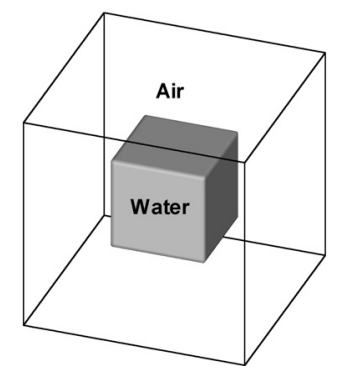

Fig. 4. Schematic models at the initial state of oscillating droplet. (a) Two-dimensional case; (b) three-dimensional case.

\subsection{Oscillating Droplet in a Zero-gravity Field}

Figure 4 shows the schematic models for oscillating droplets. The upper one indicates a two-dimensional case, and the lower one is a three-dimensional case. The initial shape of droplet is square or cubic respectively. The droplets start to oscillate due to surface tension in a zerogravity field.

Figure 5 shows a period of oscillation of the two-dimensional case. The gray part indicates the liquid while the white part indicates the air. The black arrows indicate the velocity vectors. At $\tau=0.0180$, the flow around the droplet is almost stagnant, which means that the surface energy is a maximum at this time instance then this energy is about to be transformed to the kinetic energy. As shown in the third figure, the magnitude of velocity is large when the shape of droplet is almost circular, i.e. the droplet occupies the least surface area. However, both the surface energy and the kinetic energy are gradually losing due to viscous dissipation and finally the droplet forms a perfectly round shape. The process of energy loss is more clearly presented in the next 


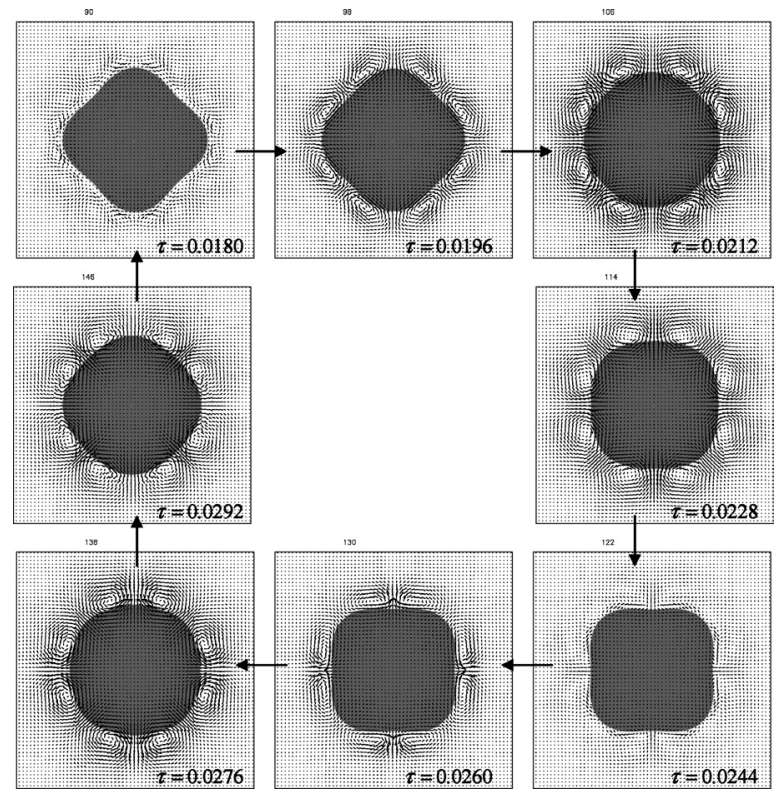

Fig. 5. Two-dimensional oscillating droplet during one period for $G=0, \Gamma=5 \times 10^{5}$, density ratio $=833$, and viscosity ratio $=56$. The vectors indicate velocity fields. The number of meshes is $128 \times 128$. The time interval $\Delta \tau=$ 0.0016 .

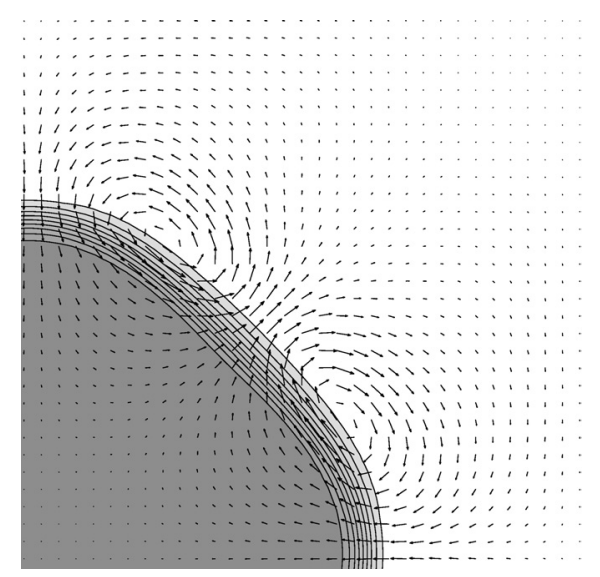

Fig. 6. A detailed profile of the two-dimensional oscillating droplet for $G=0, \Gamma=5 \times 10^{5}$, density ratio $=833$, and viscosity ratio $=56$. The vectors indicate velocity fields. The number of meshes is $128 \times 128$. The non-dimensional time instance is at $\tau=0.02$.

three-dimensional case. Figure 6 shows a detailed profile of the two-dimensional droplet at a time instant. The lines represent the iso-contours of the index function. The number of lines is 9, which indicates the interval of the neighboring values is 0.1 . Usually the liquid-gas interface tends to be less clear-cut gradually as time evolves due to numerical diffusion. However, owing to employing Eq. (22), the thickness of the transitional interface is maintained fairly well. Two vortices generate during the oscillation in this domain. It is noted that the center of a vortex is deviated in the side of gas phase because the gas density is much smaller than the liquid density.

Figure 7 shows the attenuation of the three-dimensional oscillating droplet. The number of meshes used in this simulation is $80 \times 80 \times 80$. After one period passed from the initial state, the shape of the droplet becomes a quasi-hexahe-

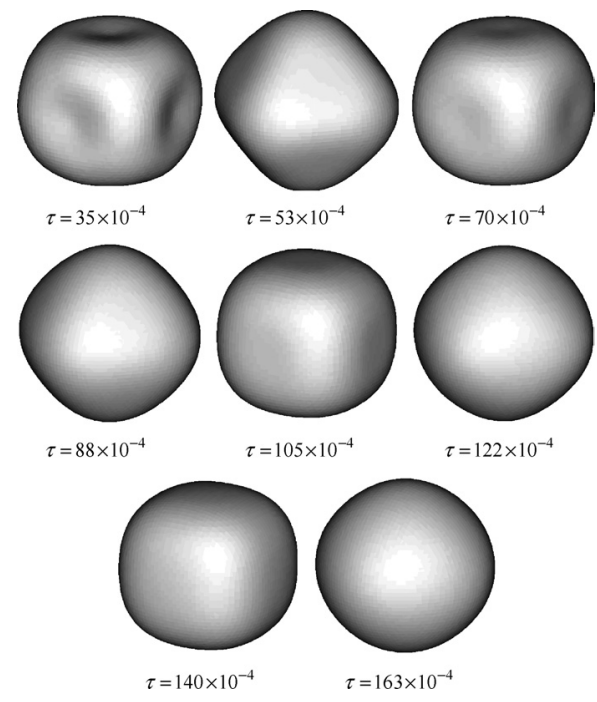

Fig. 7. Attenuating oscillation of an initially cubic droplet for $G=0, \quad \Gamma=5 \times 10^{5}$, density ratio $=100$, and viscosity ratio $=10$. The number of meshes is $80 \times 80 \times 80$.

dron shape at $\tau=35 \times 10^{-4}$. This shape is similar to the initial state but all the edges and vertices are rounded. After a further half period, the droplet becomes a quasi-octahedron shape at $\tau=53 \times 10^{-4}$. Thereafter the droplet repeats such a kind of oscillation with decaying its energy. At $\tau=$ $163 \times 10^{-4}$, the shape seems almost spherical.

\subsection{Falling Droplet in the Presence of Static Magnetic Fields}

In this subsection, dynamics of an initially-cubic droplet in the presence of both a gravity and a uniform static magnetic field is presented. Figure 8 shows a sequence of a falling droplet onto a flat wall in the presence of a uniform horizontal magnetic field. At the beginning of computation $\left(\tau=6 \times 10^{-4}\right.$ ), all the edges and vertices are rapidly deformed due to surface force. Then, the droplet is gradually accelerated due to the gravity. In the meantime, the effect of the horizontal magnetic field commences to appear as shown at $\tau=12 \times 10^{-4}$ and thereafter. The droplet shape and the resulting flow pattern are symmetric with respect to both two vertical central planes parallel and perpendicular to the magnetic field. As time evolves, tendency of two-dimensional flow becomes remarkable since the momentum of flow is gradually weakened and thus the electromagnetic force becomes conspicuous. In the flow within a cavity in the presence of a magnetic field, the electric conductivity of wall is responsible for the induced electric field in the liquid metal and hence the velocity field is also influenced by the value of wall conductance. ${ }^{14,15)}$ In this simulation, the enclosure wall is supposed to be electrically insulating and the electric current is forced to flow through the Hartmann layers. As reported in the references, ${ }^{16,17)}$ the lack of number of meshes within the Hartmann layer leads to inaccuracy of the MHD flow analysis when the Hartmann layers become active especially for the high $\mathrm{Ha}$ number. Anyway, sufficient number of meshes along the applied magnetic field is required for the simulation of MHD free-surface flow.

Figure 9 shows a series of a falling droplet onto a flat plate in the presence of a uniform vertical magnetic field. 


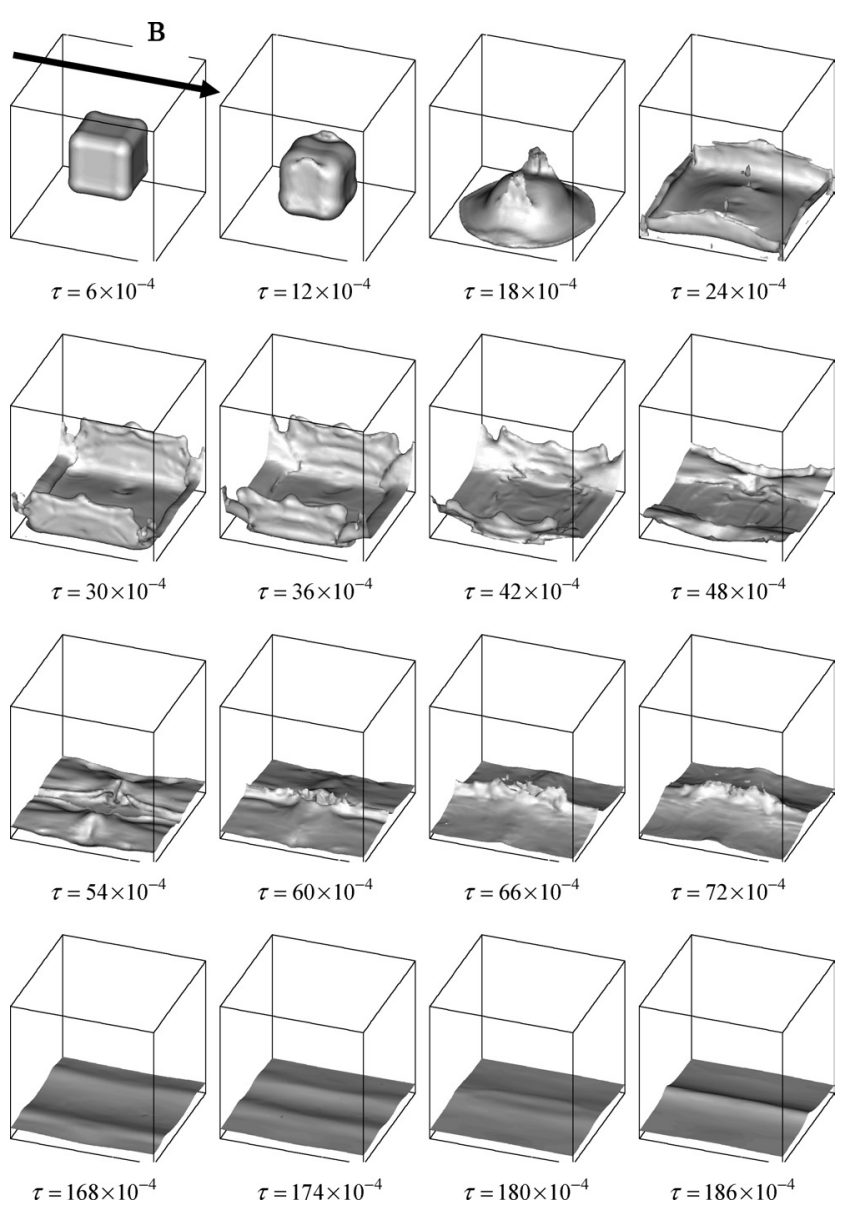

Fig. 8. A falling droplet onto a flat wall for $H a=200$, $G=1.0 \times 10^{6}, \Gamma=5 \times 10^{5}$, density ratio $=100$, viscosity ratio $=10$ and conductivity ratio $=0$. A uniform horizontal magnetic field is imposed. The number of meshes is $80 \times 80 \times 80$.

The strength of the magnetic field is the same as the case in Fig. 8. During falling before landing at the bottom wall, the falling speed is hardly influenced by the electromagnetic force since the directions of the movement of droplet and the gravity are parallel to each other. The influenced portions are the edges and vertices caused by the surface tension. After having landed at the bottom wall, the droplet gradually spreads in a symmetrical manner with respect to the central position of the bottom wall. When there are outgoing flows from the central position of the bottom wall, the electric current circulates in a clockwise direction and the resulting electromagnetic force strongly opposes the outward flows. Thus, the electromagnetic force acts to dampen the flow efficiently since the electric current is not enforced to pass through the Hartmann layer that is formed in the vicinity of the bottom wall. At $\tau=72 \times 10^{-4}$, the flow is almost suppressed. This situation is quite different from the case that the magnetic field is applied in a horizontal direction (compare with Fig. 8).

\subsection{Collapse of a Liquid Metal Column in the Pres- ence of Uniform Magnetic Fields}

Figure 10 shows the schematic model for collapse of a liquid metal column in the presence of a magnetic field. The uniform magnetic field is applied either in the longitudinal, transverse or vertical direction respectively. Figure

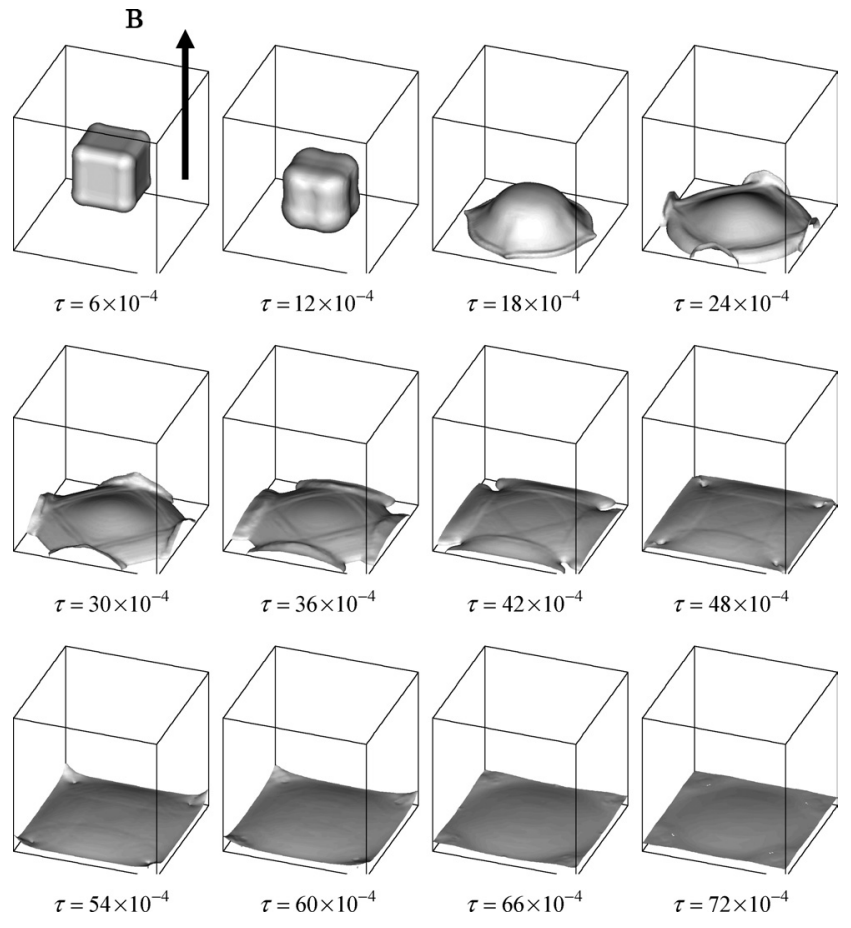

Fig. 9. A falling droplet onto a flat wall for $H a=200$, $G=1.0 \times 10^{6}, \Gamma=5 \times 10^{5}$, density ratio $=100$, viscosity ratio $=10$ and conductivity ratio $=0$. A uniform vertical magnetic field is imposed. The number of meshes is $80 \times 80 \times 80$.

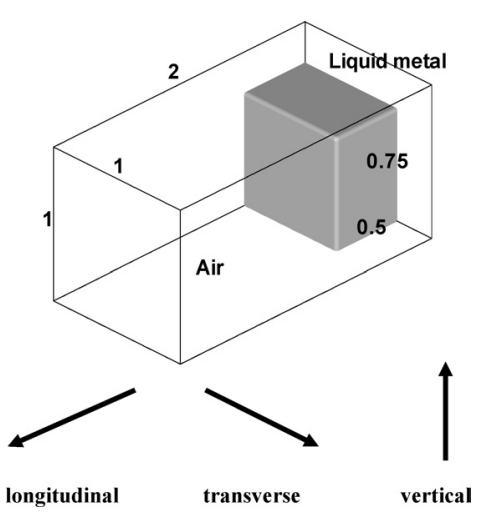

Fig. 10. Schematic model for the collapse of a liquid metal column in the presence of a uniform static magnetic field.

11 shows a series of collapse of the liquid metal column for four cases, which are without and with the longitudinal, transverse and vertical magnetic fields. The number of meshes employed in this simulation is $120 \times 60 \times 60$ and the $\mathrm{Ha}$ number is fixed at 200. Since the initial location of the column is at a corner in the rectangular enclosure, the liquid metal column collapses mostly in two directions (longitudinal and transverse directions). When horizontal magnetic fields are applied (see the second and third columns), during spreading before arriving at any vertical walls, the rate of spreading flow is almost the same as that in the case of no-magnetic field. But, after having reached to vertical walls, the rate of ascending flow is influenced by the horizontal magnetic fields. For example, in the case of longitudinal magnetic field, the ascending velocity along the vertical wall perpendicular to the applied magnetic field is slowed down. In the same reason, in the case of transverse 


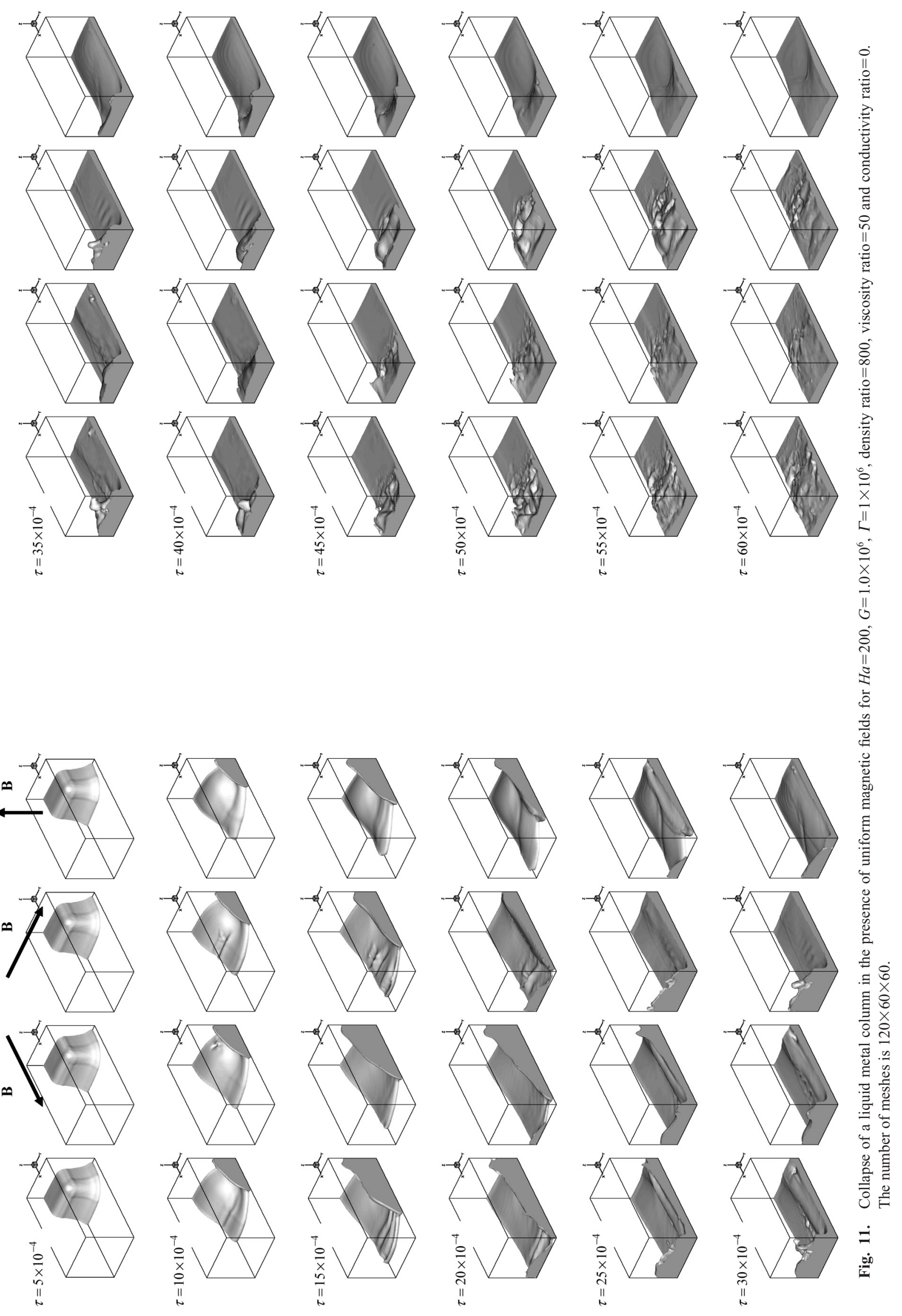


magnetic field, the ascending velocity along the vertical wall perpendicular to the applied magnetic field is weakened due to the electromagnetic force induced in the liquid metal. On the other hand, when the vertical magnetic field is applied (see the fourth column), the spreading rate is obviously slowed down by the electromagnetic force. By comparing these cases, it is concluded that the imposition of the vertical magnetic field is useful to stabilize and weaken this kind of free-surface flow, while that of the horizontal magnetic fields does not have a powerful influence on the freesurface flow but the flow will align along the magnetic field after a long time.

\section{Conclusions}

Three-dimensional numerical simulations have been carried out for several problems with a new-developed numerical modeling of MHD free-surface flows. In this paper, emphases are put on the modeling of surface force, simulation of high-density ratio and the effect of applied magnetic field. Concerning the modeling of surface force, owing to the use of CSF model, the numerical simulations have been successfully performed for the collapsing liquid column and the oscillating droplet with the density ratio up to 833 . Therefore, actual water-air two-phase simulations are possible to implement with the use of the present modeling. The effect of the uniform static magnetic field on the liquid metal free-surface flow significantly depends on the direction of the applied magnetic field. The uniform vertical magnetic field acts to dampen the flow efficiently while the uniform horizontal magnetic field acts to enforce a tendency of two-dimensional flow.

\section{Nomenclature}

$a$ : Width of initial water column (m)

$b_{0}$ : Imposed magnetic field strength

$\vec{B}$ : Dimensionless magnetic flux density

$d$ : Characteristic length $(\mathrm{m})$

$\vec{e}_{\mathrm{n}}$ : Unit vector normal to interface

$\vec{f}_{\overrightarrow{\mathrm{em}}}:$ Electromagnetic force $\left(\mathrm{N} / \mathrm{m}^{3}\right)$

$\vec{f}_{\mathrm{sa}}$ : Surface force normal to interface $\left(\mathrm{N} / \mathrm{m}^{2}\right)$

$\vec{f}_{\mathrm{sv}}:$ Volume force normal to interface $\left(\mathrm{N} / \mathrm{m}^{3}\right)$

$\vec{g}$ : Gravitational acceleration vector $\left(\mathrm{m} / \mathrm{s}^{2}\right)$

$g$ : Gravitational acceleration $\left(\mathrm{m} / \mathrm{s}^{2}\right)$

$G: \quad$ Galilei number $=g \rho_{\mathrm{G}}^{2} d^{3} / \mu_{\mathrm{G}}^{2}$

$\mathrm{Ha}$ : Hartmann number $=\sqrt{\sigma_{\mathrm{L}} / \mu_{\mathrm{L}}} b_{0} d$

$\vec{j}$ : Electric current density $\left(\mathrm{A} / \mathrm{m}^{2}\right)$

$\vec{J}$ : Dimensionless electric current density

$p:$ Pressure $(\mathrm{Pa})$

$P:$ Dimensionless pressure

$t$ : Time (s)

$\vec{u}$ : Velocity vector $(\mathrm{m} / \mathrm{s})$

$u$ : $X$-directional component of velocity $(\mathrm{m} / \mathrm{s})$

$\vec{U}$ : Dimensionless velocity vector

$U$ : Dimensionless $X$-directional component of velocity

$v: \quad Y$-directional component of velocity $(\mathrm{m} / \mathrm{s})$

$V$ : Dimensionless $Y$-directional component of velocity

$w$ : Z-directional component of velocity $(\mathrm{m} / \mathrm{s})$

$W$ : Dimensionless $Z$-directional component of velocity

$x$ : Coordinate $(\mathrm{m})$
$X:$ Dimensionless coordinate

$y:$ Coordinate $(\mathrm{m})$

$Y$ : Dimensionless coordinate

$z:$ Coordinate $(\mathrm{m})$

$Z$ : Dimensionless coordinate

Greek letters

$\gamma:$ Surface tension $(\mathrm{N} / \mathrm{m})$

$\Gamma: \quad$ Dimensionless tension number $=\gamma \rho_{\mathrm{G}} d / \mu_{\mathrm{G}}^{2}$

$\kappa$ : Curvature of interface $\left(\mathrm{m}^{-1}\right)$

$\mu:$ Viscosity (Pas)

$\mu_{\phi}:$ Dimensionless viscosity $=\mu / \mu_{\mathrm{G}}$

$\bar{\mu}: \quad$ Viscosity ratio $=\mu_{\mathrm{L}} / \mu_{\mathrm{G}}$

$\rho:$ Density $\left(\mathrm{kg} / \mathrm{m}^{3}\right)$

$\rho_{\phi}:$ Dimensionless density $=\rho / \rho_{\mathrm{G}}$

$\hat{\rho}:$ Density ratio $=\rho_{\mathrm{L}} / \rho_{\mathrm{G}}$

$\sigma$ : Electric conductivity, $\left(\Omega^{-1} \mathrm{~m}^{-1}\right)$

$\sigma_{\phi}:$ Dimensionless electric conductivity $=\sigma / \sigma_{\mathrm{L}}$

$\widetilde{\sigma}:$ Electric conductivity ratio $=\sigma_{\mathrm{G}} / \sigma_{\mathrm{L}}$

$\tau$ : Dimensionless time

$\phi:$ Index function

$\psi$ : Electric potential (V)

$\Psi$ : Dimensionless electric potential

Notations

$$
\begin{array}{cc}
\vec{\nabla}: & \partial / \partial x \cdot \vec{e}_{x}+\partial / \partial y \cdot \vec{e}_{y}+\partial / \partial z \cdot \vec{e}_{z} \text { or } \\
& \partial / \partial X \cdot \vec{e}_{x}+\partial / \partial Y \cdot \vec{e}_{y}+\partial / \partial Z \cdot \vec{e}_{z} \\
\nabla^{2}: & \partial^{2} / \partial x^{2}+\partial^{2} / \partial y^{2}+\partial^{2} / \partial z^{2} \text { or } \\
& \partial^{2} / \partial X^{2}+\partial^{2} / \partial Y^{2}+\partial^{2} / \partial Z^{2}
\end{array}
$$

Subscripts

G: Gas phase

L: Liquid phase

\section{REFERENCES}

1) H. Yasuda, I. Ohnaka, Y. Ninomiya, R. Ishii, S. Fujita and K. Kishio: J. Cryst. Growth, 260 (2004), 475.

2) C. W. Hirt and B. D. Nichols: J. Comput. Phys., 39 (1981), 201.

3) M. Sussman, P. Smereka and S. Osher: J. Comput. Phys., 114 (1994), 146.

4) F. H. Harlow and J. P. Shannon: J. Appl. Phys, 38 (1967), 3855.

5) T. Inamuro, T. Ogata, S. Tajima and N. Konishi: J. Comput. Phys., 198 (2004), 628.

6) Y.-S. Hwang, P.-R. Cha, H.-S. Nam, K.-H. Moon and J.-K. Yoon: ISIJ Int., 37 (1997), No. 7, 659.

7) D. Gao, N. B. Morley and V. Dhir: Trans. ASME J. Fluids Eng., 126 (2004), 120.

8) T. Tagawa: ISIJ Int., 45 (2005), No. 7, 954.

9) T. Yabe, T. Utsumi and Y. Ogata: CIP Method (Japanese), Morikita Shuppan Co., Ltd., Tokyo, (2003), 35.

10) J. U. Brackbill, D. B. Kothe and C. Zemach: J. Comput. Phys., 100 (1992), 335.

11) C. W. Hirt, B. D. Nichols and N. C. Romero: Los Alamos Scientific Laboratory Report, LA-5852, (1975).

12) T. Tagawa and H. Ozoe: Numer. Heat Transfer A, 30 (1996), 271.

13) N. Takada, M. Misawa and A. Tomiyama: Math. Comp. Sim., 72 (2006) 220.

14) T. Tagawa and H. Ozoe: Int. J. Heat Mass Transfer, 41 (1998), 1917.

15) L. Bühler: Phys. Fluids, 10 (1998), 223.

16) T. Tagawa, G. Authié and R. Moreau: Eur. J. Mech. B/Fluids, 21 (2002), No. 4, 383.

17) G. Authié, T. Tagawa and R. Moreau: Eur. J. Mech. B/Fluids, 22 (2003), No. 3, 203. 